\section{VIABILIDAD Y GERMINACIÓN DE SEMILLAS DE SEIS ESPECIES DE Tillandsia (BROMELIACEAE) DE OAXACA, MÉXICO}

\section{SEED VIABILITY AND GERMINATION OF SIX SPECIES OF Tillandsia (BROMELIACEAE) FROM OAXACA, MÉXICO}

\author{
Diana Sosa-Luría ${ }^{1}$, José L. Chávez-Servia ${ }^{1 \star}$, Demetria \\ Mondragón-Chaparro ${ }^{1}$, Julio A. Estrada-Gómez ${ }^{2}$ y \\ Porfirio Ramírez-Vallejo ${ }^{2}$
}

${ }^{1}$ CIIDIR-Unidad Oaxaca, Instituto Politécnico Nacional. Hornos 1003, Santa Cruz Xoxocotlán. 71230, Oaxaca, México. ${ }^{2}$ Posgrado en Recursos Geneticos y Productividad-Genética, Colegio de Postgraduados. Km. 36.5 Carr. México-Texcoco. 56230, Montecillo, Texcoco, Estado de México.

*Autor para correspondencia (jchavezs@ipn.mx)

\section{RESUMEN}

Para diseñar estrategias de conservación ex situ e in situ de los acervos genéticos mexicanos de las especies nativas de Tillandsia, es fundamental un mejor entendimiento de la ecofisiología de la semilla. Por ello, en este trabajo se evaluó la viabilidad y capacidad germinativa de la semilla de seis especies del género Tillandsia (Bromeliaceae). La semilla se recolectó de febrero a julio de 2010 en bosques de Santa Catarina Ixtepeji, Oaxaca. La viabilidad se midió mediante la prueba de rayos $\mathrm{X}$ y la germinación en una incubadora a temperatura constante de $25^{\circ} \mathrm{C}$ y fotoperiodo neutro. En ambas pruebas los tratamientos se distribuyeron en un diseño completamente al azar con un mínimo de seis repeticiones de 50 semillas. Los resultados se analizaron con comparación múltiple de medias y una correlacion de Pearson. Hubo diferencias $(P \leq 0.05)$ entre especies para porcentaje de semillas completas (viables o completamente desarrolladas) y de semillas con desarrollo incompleto. T. violacea presentó los más bajos porcentajes de semillas completas $(61.3 \%)$ y altos porcentajes de semillas con desarrollo parcial (31.6\%), en comparación con T. prodigiosa, T. carlos-hankii, $T$. bourgaei, $T$. makoyana y $T$. fasciculata que presentaron entre 68.3 y 89.0 $\%$ de semillas completas y entre 4.5 y $15.0 \%$ de semillas parcialmente desarrolladas. $T$. bourgaei presentó el mayor porcentaje en su capacidad germinativa (83.3\%), seguida de T. makoyana (79.8\%), T. carlos-hankii (79.5 \%), T. prodigiosa (75.8 \%), T. fasciculata (45.0 \%) y T. violacea (15.2\%). Hubo correlación positiva y significativa $(r=0.69, \mathrm{P}<0.05)$ entre los porcentajes de semillas completas y semillas germinadas.

Palabras clave: Tillandsia, poblaciones silvestres, rayos X, tasa de germinación.

\section{SUMMARY}

Understanding the seed ecophysiology of native species of Tillandsia is crucial to design ex situ and in situ conservation strategies for Mexican gene pools. In the present work, seed viability and germination capability of six species of the Tillandsia (Bromeliaceae) genus were evaluated using seed collected from February to July 2010, at Santa Catarina Ixtepeji, Oaxaca forest. All seed lots were evaluated by X-rays and germination tests (at constant temperature of $25^{\circ} \mathrm{C}$ and neutral photoperiod), both tests under a completely random design with a minimum of 6 replicates of 50 seeds each. Significant differences $(P<0.05)$ were observed among Tillandsia species in relation to full or complete (viable or structures completely developed) and incomplete seed percentages. T. violacea presented the lower percentage of full seeds $(61.3$ $\%)$ and higher percentage of seeds with structures partially developed (31.6\%), compared to T. prodigiosa, T. carlos-hankii, T. bourgaei, $T$. makoyana and $T$. fasciculate which showed values between 68.3 and $89 \%$ of full seeds and 4.5 to $15.0 \%$ of incomplete seeds. T. bourgaei presented the highest percentage of germination capability $(83.3 \%)$, followed by T. makoyana (79.8\%), T. carlos-hankii (79.5\%), T. prodigiosa (75.8 \%), T. fasciculata (45.0 \%) and $T$. violacea (15.16\%). A positive and significant correlation $(r=0.69, P<0.05)$ was determined between the percentages of full seed from the $\mathrm{X}$-rays test and germinated seeds in the germination test.

Index words: Tillandsia, wild populations, X-rays, germination rate.

\section{INTRODUCCIÓN}

Un ejemplo de la biodiversidad en México es la familia Bromeliaceae la cual incluye 18 géneros que agrupan a 342 especies. En particular, el género Tillandsia es el más abundante ya que posee 192 especies, 133 de ellas son endémicas y están ampliamente distribuidas en diversas condiciones ambientales y de vegetación (Espejo et al., 2004; Espejo-Serna et al., 2007).

En México, diferentes especies de la esta familia se encuentran en riesgo de extinción como consecuencia de la pérdida de la vegetación, ya que más de la tercera parte de los bosques y selvas han sido deforestados (Ricker et al., 2007), y por la extracción ilegal (Flores-Palacios y Valencia-Díaz 2007; Mondragón, 2009), entre otras causas. En las normas oficiales mexicanas NOM-059-SEMARNAT-2002 y NOM-059-SEMARNAT-2010, 21 especies de bromelias endémicas se enlistan en las categorías de amenazadas y sujetas a protección especial.

Ante este riesgo, una estrategia inmediata es su conservación ex situ en bancos de germoplasma o la conservación in situ en zonas oficialmente declaradas como "reserva", complementada con acciones que eviten la deforestación de bosques donde se localizan las poblaciones endémicas y la extracción ilegal de individuos de dichas especies. En cualquier caso, las estrategias o acciones a favor de la conservación deben partir del entendimiento de la dinámica de las poblaciones silvestres. En particular, para implementar una estrategia de conservación ex situ y el manejo eficiente del germoplasma en un banco, se necesita información básica de las características fisiológicas de las semillas y de las condiciones óptimas de almacenamiento (Engels y Visser, 2003).

Las semillas del género Tillandsia miden menos de 5 $\mathrm{mm}$; poseen cubierta semiplumosa, endospermo, un cotiledón, vestigios de hojas primarias y secundarias, ápice del brote, e hipocotilo. El embrión llena sólo un tercio del volumen total y siempre ocupa la posición opuesta al punto de inserción de la pared del ovario. Contienen un apéndice 
epidérmico plumoso, por lo que su dispersión es anemócora. Cada semilla se inserta, mediante su apéndice, en una cápsula dehiscente que al abrirse las transporta por el aire. El apéndice funciona como "adherente" a los troncos y ramas de los árboles, y permite la absorción de agua (Esteba, 2002; Wester y Zotz, 2011).

En la familia Bromeliacea existe información acerca de la demografía de las poblaciones silvestres, morfología de plantas y mecanismos de dispersión de semillas. Sin embargo, son pocos los trabajos relacionados con la capacidad germinativa y los factores determinantes de la viabilidad de sus semillas. Los principales géneros estudiados, en aspectos de semillas, son: Dyckia (Pompelli et al., 2006; Vieira et al., 2007; Duarte et al., 2010); Encholirium (Tarré et al., 2007); Alcantarea, Pitcairnia y Vriesea (Pereira et al., 2009; Pereira et al., 2010a); Aechmea (Pinheiro y Borghetti, 2003); Puya (Vadillo et al., 2004); Pseudananas (Vieira y Silveira, 2010); Nidularium (Pereira et al., 2010b); Tillandsia (Mondragón y Calvo-Irabíen, 2004; Montes-Recinas et al., 2012) y Catopsis (Palací et al., 2004; Wester y Zotz, 2011). En las especies endémicas mexicanas de Tillandsia es escasa o nula la información disponible sobre semillas.

El objetivo de este trabajo fue evaluar la viabilidad y germinación de semillas de seis especies silvestres de Tillandsia L. distribuidas en los bosques de Santa Catarina Ixtepeji, Oaxaca.

\section{MATERIALES Y MÉTODOS}

\section{Recolecta de material biológico}

Se seleccionaron cinco especies endémicas de Tillandsia (T. carlos-hankii, T. prodigiosa, T. bourgaei, T. violacea, y T. makoyana) y T. fasciculata, todas reportadas con algún tipo de uso (Mondragón y Villa, 2008; Mondragón, 2009), que se distribuyen en los bosques templados de Santa Catarina Ixtepeji, Oaxaca. Esta región forma parte de la Sierra Norte de Oaxaca, México. En la zona de colecta el clima varía de semicálido-subhúmedo con lluvias en verano a semifríosubhúmedo con lluvias en verano; la isoterma media anual oscila entre 11 y $16^{\circ} \mathrm{C}$; y la precipitación media anual varía de 600 a $1300 \mathrm{~mm}$ (García, 2004). La recolecta de semillas se hizo de febrero a junio de 2010. En el Cuadro 1 se presenta una descripción general de la localización y características de inflorescencias y de semillas colectadas.

Muestreo de semillas. Una vez que las poblaciones se ubicaron, al ocurrir la dispersión de semillas se recolectaron tres o cuatro cápsulas de color café-marrón de 50 individuos por población y por especie localizadas en un radio de $500 \mathrm{~m}$, con base en los criterios propuestos por Hay y Smith (2004). Debido a la naturaleza epífita del género, cada individuo muestreado se encontraba en fororito distinto. En el caso de T. fasciculata y T. violacea, se recolectaron semillas de algunos individuos en proceso de dispersión, debido a la escasa disponibilidad de cápsulas cerradas en esas poblaciones. Las cápsulas se secaron a la sombra en un lugar seco, fresco y ventilado $\left(13\right.$ a $25^{\circ} \mathrm{C}$ y 20 a $40 \%$ de humedad relativa) durante cuatro semanas, y una vez que las cápsulas abrieron se extrajo la semilla.

\section{Evaluaciones de viabilidad y capacidad germinativa}

Las pruebas de viabilidad y germinación se efectuaron en el Laboratorio de Semillas del Banco de Germoplasma de la Secretaría de Desarrollo Agropecuario, Forestal y Pesca del Gobierno del Estado de Oaxaca, México.

La viabilidad o calidad física de la semilla de las seis especies de Tillandsia se evaluó con la técnica de rayos X, en una muestra aleatoria de 200 semillas visualmente identificadas como sanas. Se empleó un diseño experimental completamente al azar, con cuatro repeticiones de 50 semillas cada una. Las semillas se pegaron a una placa de plástico autoadherible de polipropileno, la que se colocó sobre una placa de acrílico transparente en la cámara de irradiación no digital (Faxitron X-Ray modelo MX-20; Specimen Radiography System ${ }^{\circledR}$, Illinois, USA) y se sometió a exposiciones a $18 \mathrm{kV}$ por $10 \mathrm{~s}$. Posteriormente, las placas se revelaron en una impresora digital para rayos $\mathrm{X}$ (procesador Hope $\mathrm{X}$ Ray; Micro-Max modelo 319®). Finalmente, en cada placa radiográfica se aplicó los criterios sugeridos por Dos Santos et al. (2009) para contar: (i) semillas sanas (con estructuras completamente desarrolladas), (ii) semillas parcialmente desarrolladas (semillas con daño estructural o malformadas), y (iii) semillas vanas ( lladas ). Las variables se expresaron en porcentaje.

La capacidad germinativa de las semillas de T. bourgaei, T. prodigiosa, T. carlos-hankii y T. makoyana se evaluó en un diseño completamente al azar con 12 repeticiones de 50 semillas cada una y con seis repeticiones de igual número de semillas en T. violacea, y T. fasciculata. Esto último debido a la baja disponibilidad de semillas durante la colecta. Las semillas de cada repetición se distribuyeron en una caja Petri de vidrio de $10 \mathrm{~cm}$ de diámetro previamente esterilizada, que contenía papel filtro al que se agregó $4 \mathrm{~mL}$ de agua destilada. Las cajas Petri se colocaron en una cámara de germinación (Light-Dark Seedburo ${ }^{\circledR}$ ) con fotoperiodo neutro (12 horas luz y12 horas obscuridad) y temperatura constante de $25^{\circ} \mathrm{C}$.

Una vez germinadas las primeras semillas, lo cual ocurrió $3 \mathrm{~d}$ después de la siembra ( $\mathrm{dds}$ ), cada 24 h se contaron las semillas germinadas (con elongación del hipocotilo visible a simple vista) durante $20 \mathrm{~d}$ consecutivos hasta que la 
Cuadro 1. Descripción general de la distribución, morfología y recolecta de semilla de seis especies de Tillandsia.

\begin{tabular}{|c|c|c|c|}
\hline \multirow{2}{*}{ Descriptor general } & \multicolumn{3}{|c|}{ Especie de Tillandsia $^{\dagger}$} \\
\hline & carlos-hankii & makoyana & prodigiosa \\
\hline $\begin{array}{l}\text { Hábitat o vegetación asociada } \\
\text { (distribución) }\end{array}$ & $\begin{array}{c}\text { Pino-encino } \\
(1900-2900 \mathrm{msnm})\end{array}$ & $\begin{array}{l}\text { Pino-encino } \\
(50-2320 \mathrm{msnm})\end{array}$ & $\begin{array}{c}\text { Pino-encino } \\
(1800-2800 \mathrm{msnm})\end{array}$ \\
\hline Características de la inflorescencia & $\begin{array}{l}\text { Cilíndrica de } 57 \text { a } 70 \mathrm{~cm} \\
\text { de long., corola verde }\end{array}$ & $\begin{array}{l}\text { Erecta paniculada de hasta } \\
1 \mathrm{~m} \text {, flores violetas }\end{array}$ & $\begin{array}{l}\text { Tipo péndula, bipinnada, } \\
\text { subcilíndrica, } 30 \text { a } 60 \mathrm{~cm} \text {, } \\
\text { corola verde cremoso }\end{array}$ \\
\hline Flores por eje floral (aprox.) & 70 & 25 & 30 \\
\hline Semillas por cápsula (aprox.) & 200 a 250 & 80 a 100 & 180 a 200 \\
\hline Época de dispersión de semillas & Noviembre-Abril & Noviembre-Abril & Noviembre-Marzo \\
\hline $\begin{array}{l}\text { Paraje de origen de semillas en } \\
\text { Santa Catarina Ixtepeji }\end{array}$ & $\begin{array}{c}\text { Petenera: } 17.12281^{\circ} \\
\text { LN, } 96.3525^{\circ} \text { LO y } 2520 \\
\text { msnm }\end{array}$ & $\begin{array}{c}\text { Xhia: } 17.16389^{\circ} \mathrm{LN}, \\
96.31515^{\circ} \mathrm{LO} \text { y } 1846 \\
\mathrm{msnm}\end{array}$ & $\begin{array}{c}\text { El Vivero: } 17.1304^{\circ} \mathrm{LN} \\
96.57199^{\circ} \mathrm{LO} \text { y } 2404 \\
\mathrm{msnm}\end{array}$ \\
\hline \multirow[t]{3}{*}{ Fecha de recolecta (dd/mm/aaaa) } & $20 / 02 / 2010$ & $07 / 03 / 2010$ & $08 / 03 / 2010$ \\
\hline & \multicolumn{3}{|c|}{ Especie de Tillandsia ${ }^{\dagger}$} \\
\hline & bourgaei & fasciculata & violacea \\
\hline $\begin{array}{l}\text { Hábitat o vegetación asociada } \\
\text { (distribución) }\end{array}$ & $\begin{array}{l}\text { Encino-pino }(600-3100 \\
\text { msnm) }\end{array}$ & $\begin{array}{l}\text { Encino y selvas bajas } \\
(1500-2000 \mathrm{msnm})\end{array}$ & $\begin{array}{l}\text { Encino-pino, mesófilo de } \\
\text { montaña, pino-encino } \\
(600-3100 \mathrm{msnm})\end{array}$ \\
\hline Características de la inflorescencia & $\begin{array}{l}\text { Erecta, cilíndrica, } 26 \text { a } \\
30 \mathrm{~cm} \text {, corola verde o } \\
\text { amarillo verduzco }\end{array}$ & $\begin{array}{l}\text { Paniculada, } 25 \text { a } 60 \mathrm{~cm} \text { de } \\
\text { long., corola violeta }\end{array}$ & $\begin{array}{l}\text { Inflorescencia colgante, } 15 \\
\text { a } 35 \mathrm{~cm} \text { de long., corola } \\
\text { tubular violeta }\end{array}$ \\
\hline Flores por eje floral (aprox.) & 30 & 8 & 10 \\
\hline Semillas por cápsula (aprox.) & 200 a 250 & 80 a 120 & 200 a 250 \\
\hline Época de dispersión de semillas & Marzo-Mayo & Mayo-Julio & Marzo-Junio \\
\hline $\begin{array}{l}\text { Paraje de origen de semillas en } \\
\text { Santa Catarina Ixtepeji }\end{array}$ & $\begin{array}{l}\text { El Cerezal: } 17.1596^{\circ} \mathrm{LN} \\
96.3589^{\circ} \mathrm{LO} \text { y } 2245 \mathrm{msnm}\end{array}$ & $\begin{array}{l}\text { Xhia: } 17.18072^{\circ} \mathrm{LN} \\
96.31359^{\circ} \mathrm{LO} \text { y } 1514 \\
\text { msnm }\end{array}$ & $\begin{array}{l}\text { Peña Prieta: } 17.09413^{\circ} \\
\text { LN, 96.38103º LO y } 2835 \\
\text { msnm }\end{array}$ \\
\hline Fecha de recolecta (dd/mm/aaaa) & 09/03/2010 & 03/03/2010 y $19 / 07 / 2010$ & $20 / 02 / 2010$ y $27 / 06 / 2010$ \\
\hline
\end{tabular}

†Fuentes: Smith y Downs (1974), Espejo-Serna et al. (2007), Mondragón y Villa (2008), y experiencias previas de los autores.

germinación se estabilizó. Con esta información se estimó el porcentaje de germinación total, la tasa de germinación $\left[\mathrm{TG}=\sum\right.$ (núm. semillas germinadas al i-ésimo día/tiempo en días a la i-ésima evaluación)] y el tiempo medio a $50 \%$ de germinación (T50\%, en días).

\section{Análisis estadístico}

Las variables expresadas en porcentaje se transformaron mediante la expresión arcoseno $(\sqrt{ } \%)$ (Steel y Torrie, 1985) previo al análisis de varianza. La comparación múltiple de medias se hizo mediante la prueba de Tukey $(\mathrm{P} \leq 0.05)$. También se hizo un análisis de correlación simple de Pearson $(\mathrm{P} \leq 0.05)$ entre el porcentaje de semillas germinadas $\mathrm{y}$ el porcentaje de semillas sanas visualizadas en la prueba de rayos $\mathrm{X}$. Todos los análisis se hicieron con el paquete estadístico SPSS (versión 11.0 para Windows).

\section{RESULTADOS Y DISCUSIÓN}

Hubo diferencias significativas entre especies en el porcentaje de semillas sanas y semillas con embriones parcialmente desarrollados, pero no para el porcentaje de semillas vanas (Cuadro 2). La presencia de un alto porcentaje de semillas con embrión parcialmente desarrolladas y vanas (entre 11 y $39.1 \%$ ), que a simple vista parecían sanas, muestran que el uso de la prueba de rayos X puede ser útil para evaluar estos atributos de semillas en la subfamilia Tillandsioidea que se caracterizan por sus tamaños pequeños $(<5 \mathrm{~mm})$.

Las imágenes radiográficas de las semillas de Tillandsia violacea mostraron que, en promedio, una alta proporción (31.6\%) no presentaban las estructuras internas 


\begin{tabular}{|c|c|c|c|}
\hline Especie & Sanas (\%) & Con embrión parcialmente desarrollado (\%) & Vacías (\%) \\
\hline T. prodigiosa & $79.4 \mathrm{a}^{\dagger}$ & $15.0 \mathrm{a}$ & $5.6 \mathrm{a}$ \\
\hline T. carlos-hankii & $89.0 \mathrm{a}$ & $4.5 \mathrm{a}$ & $6.5 \mathrm{a}$ \\
\hline T. bourgaei & $86.3 \mathrm{a}$ & $7.5 \mathrm{a}$ & $8.1 \mathrm{a}$ \\
\hline T. makoyana & $83.4 \mathrm{a}$ & $7.9 \mathrm{a}$ & $8.7 \mathrm{a}$ \\
\hline T. fasciculata & $68.3 \mathrm{a}$ & $14.0 \mathrm{a}$ & $17.7 \mathrm{a}$ \\
\hline T. violacea & $61.3 \mathrm{~b}$ & $31.6 \mathrm{~b}$ & $8.1 \mathrm{a}$ \\
\hline Promedio & 63.2 & 20.2 & 15.7 \\
\hline CV (\%) & 9.7 & 8.2 & 8.4 \\
\hline Valor de F & 4.7 & 16.5 & $1.5 \mathrm{~ns}$ \\
\hline
\end{tabular}

completamente desarrolladas (Cuadro 2). Estos resultados describen parte de las dificultades de campo en conseguir semillas de T. fasciculata y T. violacea, de las cuales fue necesario colectar semilla en dos épocas (Cuadro 1), lo que posiblemente afectó las respuestas. $\mathrm{Al}$ respecto, Scatena et al. (2006) indican que las condiciones micro-ambientales en las que se desarrolla la planta madre afectan el desarrollo o maduración de las semillas de Tillandsia. Por ejemplo, el apéndice plumoso, característico del género, puede variar en longitud, y el cotiledón puede no desarrollarse. Este efecto también es común en árboles forestales de dispersión anemócora de semillas (Schmidt, 2007).

Tillandsia prodigiosa, T. carlos-hankii, T. bourgaei, T. makoyana y T. fasciculata no difirieron significativamente en porcentaje de semillas completas, parcialmente desarrolladas y vanas (Cuadro 2). En estas especies, la suma de semillas con estructuras completas y con desarrollo parcial fue superior a $80 \%$, con amplia posibilidad de originar plántulas normales, como encontraron Machado y Cícero (2003) en Lithraea molleoides (Anacardiaceae) y Oliveira et al. (2003) en Peltophorium dubium (Caesalpiniaceae).

Las especies con mayor porcentaje de semillas con estructuras completas presentaron los mayores porcentajes de germinación (Cuadro 2, Figura 1). Todas las especies de Tillandsia iniciaron su germinación (hipocotilo visible) a partir del día 3 después de la siembra, y la estabilización de germinación total se observó a partir del día 19 en la mayoría de las especies, excepto en $T$. fasciculta y $T$. violácea donde la estabilización ocurrió a los 7 dds (Figura 1). En la bromelia Dyckia goehringii, Duarte et al. (2010) indicaron que el inicio de germinación fue a los $4 \mathrm{~d}$ y la estabilización a los 14 d; y en Aechmea bracteata fue a los 5 y 20 d, respectivamente (Goode y Allen, 2009). Los resultados aquí mostrados se ubican dentro de los rangos determinados para la familia Bromeliaceae. Cabe señalar que las semillas no germinadas se contaminaron con hongos.

El porcentaje de germinación fue diferente $(\mathrm{P} \leq 0.05)$ entre especies. La germinación de las semillas de Tillandsia prodigiosa, T. carlos-hankii, T. makoyana y T. bourgaei varió de 75.8 a $83.3 \%$, y fue mayor a la de T. fasciculata $(45.0 \%)$ y T. violacea (15.2 \%) (Cuadro 3). Esto indica que las especies con menores porcentajes de semillas sanas y altos porcentajes de semillas poco desarrolladas tuvieron menores porcentajes de germinación (Cuadros 2, 3; Figura 1), lo que se confirmó al obtenerse una correlación significativa y positiva $\left(r=0.69^{*}, \mathrm{P}<0.05\right)$ entre el porcentaje de germinación y el porcentaje de semillas con estructuras completas. La velocidad de germinación y tiempo medio de germinación también varió entre especies (Cuadro 3).

La capacidad germinativa de las cuatro mejores especies de Tillandsia aquí registradas, en general concuerda con la reportado por otros autores en estudios efectuados en condiciones de laboratorio. Por ejemplo, Benzing (1978) encontró una variación 85 a $90 \%$ en T. paucifolia. CastroHernández et al. (1999) obtuvieron valores más altos en T. guatemalensis (98 a $97.65 \%$ ) al igual que Mondragón y Calvo-Irabién (2006) en T. brachycaulos (100\%). De las seis especies evaluadas, $T$. violacea presentó los porcentajes más bajos (15.2\%), inferiores a los de Vadillo et al. (2004) en Puya raimondii (24.6 a $74.9 \%$ en ambiente natural) y a los de Tarré et al. (2007) en Encholirium scrutor (45\%). Las diferencias de resultados entre este trabajo y los demás podría deberse a la condición silvestre de las poblaciones aquí evaluadas, y en el caso de T. fasiculata y $T$. violaceae a las condiciones al momento de la colecta.

El paralelismo entre los resultados de la prueba de rayos $\mathrm{X}$ y los de la prueba de germinación sugiere que la capacidad 


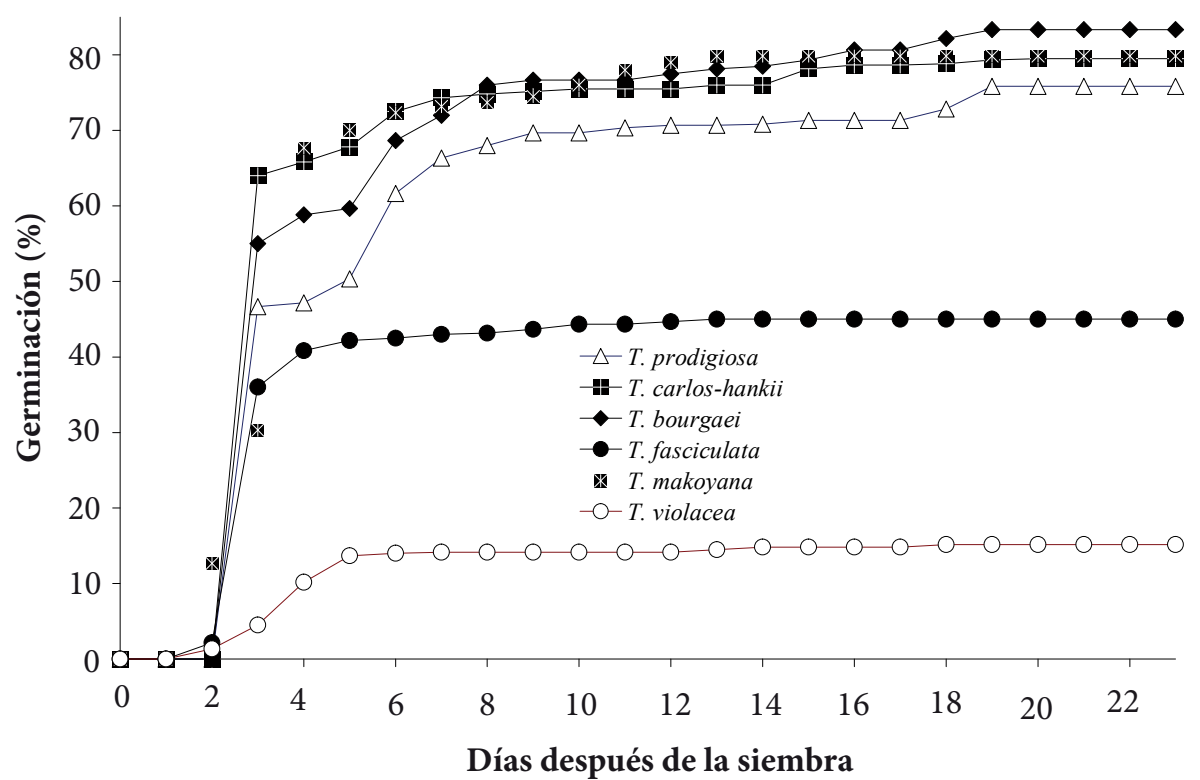

Figura 1. Porcentajes de germinación acumulada de semillas de seis especies de Tillandsia, en condiciones controladas $\left(25^{\circ} \mathrm{C},>70 \% \mathrm{HR}\right.$ y fotoperiodo neutro).

Cuadro 3. Porcentajes de germinación total, velocidad de germinación y tiempo medio a $50 \%$ de germinación (T50 \%) de semillas de Tillandsia.

\begin{tabular}{|c|c|c|c|}
\hline Especie & Germinación (\%,DE) & Velocidad de germinación & T50\% (días) \\
\hline T. prodigiosa & $75.8 \pm 5.1 \mathrm{~b}$ & $9.8 \mathrm{c}$ & $4.8 \mathrm{a}$ \\
\hline T. carlos-hankii & $79.5 \pm 6.6 \mathrm{ab}$ & $11.8 \mathrm{bc}$ & $3.0 \mathrm{~b}$ \\
\hline T. bourgaei & $83.3 \pm 5.8 \mathrm{a}$ & $11.2 \mathrm{c}$ & $3.0 \mathrm{~b}$ \\
\hline T. makoyana & $79.8 \pm 9.1 \mathrm{ab}$ & $23.2 \mathrm{a}$ & $4.0 \mathrm{ab}$ \\
\hline T. fasciculata & $45.0 \pm 7.2 \mathrm{c}$ & $14.0 \mathrm{~b}$ & --- \\
\hline T. violacea & $15.2 \pm 1.8 \mathrm{~d}$ & $4.0 \mathrm{~d}$ & --- \\
\hline Promedio & 68.6 & 14.2 & 3.7 \\
\hline CV (\%) & 6.4 & 11.9 & 10.8 \\
\hline Valor de F & $154.8^{\star *}$ & $85.9^{\star * *}$ & $1.9^{\star *}$ \\
\hline
\end{tabular}

germinativa está determinada por la madurez del embrión y la presencia de cotiledón y de apéndices plumosos, entre otros aspectos relacionados con el desarrollo completo de las estructuras de la semilla. Las Tillandsia epífitas generalmente se desarrollan en condiciones de baja disponibilidad de agua y nutrimentos, así como una reducida disponibilidad de polinizadores (Carranza-Quinceno y Estévez-Varón, 2008; Zotz y Asshoff, 2010; Zotz et al., 2010), lo cual aumenta la probabilidad de que la semilla no alcance un desarrollo completo. La valoración rápida por rayos $\mathrm{X}$ es una opción viable para determinar indirectamente la capacidad germinativa y para decidir recolectar más semilla que satisfaga la calidad mínima requerida. Por ejemplo, para ingresar un lote de semillas a un banco de germoplasma se requiere un mínimo de $75 \%$ de germinación (Engels y
Visser, 2003).

\section{CONCLUSIONES}

Hay diferencias significativas en la proporción de semillas sanas o con embriones parcialmente desarrollados así como en capacidad germinativa entre las seis especies de Tillandsia. T. violacea presentó los menores porcentajes de semillas sanas y mayores porcentajes de semillas con embriones parcialmente desarrollados, en comparación con T. prodigiosa, T. carlos-hankii, T. bourgaei, T. makoyana y T. fasciculata. En cuanto a su capacidad germinativa, $T$. bourgaei presentó el mayor porcentaje (83.3\%), seguida en orden descendente por T. makoyana, T. carlos-hankii, T. prodigiosa, T. fasciculata y T. violacea (15.16\%). El patrón de germinación se repitió en la tasa de germinación. Se 
determinó una correlación positiva y significativa entre los porcentajes de semillas completas (análisis radiográfico) y la capacidad germinativa.

\section{BIBLIOGRAFÍA}

Benzing D H (1978) Germination and early establishment of Tillandsia circinata Schlecht. (Bromeliaceae) on some of its hosts and other supports in Southern Florida. Selbyana 5:95-106.

Carranza-Quiceno J A, J V Estévez-Varón (2008) Ecología de la polinización de Bromeliaceae en el dosel de los bosques neotropicales de montaña. Bol. Cient. Mus. Hist. Nat. 12: 38-47.

Castro-Hernández J C, J H D Wolf, J G García-Franco, M GonzálezEspinosa (1999) The influence of humidity, nutrients and light on the establishment of the epiphytic bromeliad Tillandsia guatemalensis in the highlands of Chiapas, Mexico. Rev. Biol. Trop. 47:763-773.

Dos Santos S A, R F da Silva, M G Pereira, J C Machado, C F Machado, F M Borem, V M Gomes, O A O Tonetti (2009) X-ray technique application in evaluating the quality of popaya seeds. Seed Sci. Technol. 37:776-780.

Duarte E F, I F Carneiro, N F da Silva, N N R Guimarães (2010) Características físicas e geminação de sementes de Dyckia goehringii Gross \& Rauh (Bromeliaceae) sob diferentes temperaturas. Pesq. Agrop. Trop. 40:422-429.

Engels, J M M, L Visser (eds) (2003) A Guide to Effective Management of Germplasm Collections. IPGR Handbook for Genebanks No. 6. IPGR, Rome, Italy. $172 \mathrm{p}$.

Espejo-Serna A, A R López, I Ramírez, B K Holst, H E Luther. W Till (2004) Checklist of Mexican bromeliaceae with notes on species distribution and levels of endemism. Selbyana 25:33-86.

Espejo-Serna A, A R López-Ferrari, N Martínez-Correa, V A PulidoEsparza (2007) Bromelias flora de Oaxaca, México: richness and distribution. Acta Bot. Mex. 81:71-147.

Esteba F O (2002) Bromelias para Aficionados. Sociedad Venezolana de Ciencias Naturales, Comité de Bromeliología. Caracas, Venezuela. $230 \mathrm{p}$

Flores-Palacios A, S Valencia-Díaz (2007) Local illegal trade reveals unknown diversity and involves a high species richness of wild vascular epiphytes. Biol. Conserv. 136: 372-387.

García E (2004) Modificaciones al Sistema de Clasificación Climática de Köppen. Serie Libros No. 6, Instituto de Geografía, Universidad Nacional Autónoma de México, México, D.F. 90 p.

Goode L K, M F Allen (2009) Seed germination conditions and implications for establishment of an epiphyte, Aechmea bracteata (Bromeliaceae). Plant Ecol. 204:179-188.

Hay F, R D Smith (2004) Seed maturity: when to collect seed from wild plants. In: Seed Conservation: Turning Science into Practice. R D Smith, J B Dickie, S H Linington, H W Pritchard, R J Probert (eds). Royal Botanic Gardens Kew, UK. pp:99-133.

Machado C F, S M Cícero (2003) 'Aroeira-branca' (Lithraea molleoides (Vell.) Engl.-Anacardiaceae) seed quality evaluation by the Xray test. Sci. Agric. 60:393-397.

Mondragón D, L Calvo-Irabíen (2006) Seed dispersal and germination of Tillandsia brachycaulos an epiphytic plant in the Yucatán Península. South. Nat. 51:462-470.

Mondragón D, D Villa (2008) Estudio etnobotánico de las bromelias epífitas en la comunidad de Sta. Catarina Ixtepeji. Polibotánica 26:175-191.

Mondragón D (2009) Aspectos etnobotánicos de la comercialización de bromelias epifitas en Oaxaca. Rev. Etnobiol. 6:24-28

Montes-Recinas S, J Márquez-Guzmán, A Orozco-Segovia (2012) Temperature and water requeriments for germination and effects of discontinuous hydration on germinated seed survival in Tillandsia recurvata L. Plant Ecol. 213:1069-1079.

Norma Oficial Mexicana (NOM-059-SEMARNAT-2001) (2002) Diario Oficial de la Federación. Estados Unidos Mexicanos y Secretaría de Medio Ambiente y Recursos Naturales, México, D.F. 6 de marzo 2002.
Norma Oficial Mexicana (NOM-059-SEMARNAT-2010) (2010) Diario Oficial de la Federación. Estados Unidos Mexicanos y Secretaría de Medio Ambiente y Recursos Naturales, México, D.F. 15 de diciembre de 2010 .

Oliveira L M, M L M Carvalho, A C Davide (2003) Utilização do teste de raios-X na avaliação da qualidade de sementes de Canafístula Peltophorium dubium (Sprengel) Taubert. Rev. Brasil. Sementes 25:116-120.

Palací C A, G K Brown, D E Tuthill (2004) The seed of Catopsis (Bromeliaceae: Tillandsioideae). Syst. Bot. 29:518-527.

Pereira A R, A C S Andrade, T S Pereira, R C Forzza, A S Rodrigues (2009) Comportamento germinativo de espécies epífitas e rupícolas de Bromeliaceae do Parque Estadual do Ibitipoca, Minas Gerais, Brasil. Rev. Brasil. Bot. 32:827-838.

Pereira A R, A C S Andrade, T S Pereira, R C Forzza, A S Rodrigues (2010a) Morphological aspects of seed, germination and storage of Pitcairnia albiflos (Bromeliaceae). Seed Sci. Technol. 38:79-87.

Pereira C, F L Cuquel, M Panobianco (2010b) Germinação e armazenamento de sementes de Nidularium innocentii (Lem.). Rev. Brasil. Sementes 32:36-41.

Pinheiro F, F Borghetti (2003) Light and temperature requirements for germination of seeds of Aechmea nudicaulis (L.) Griesebach and Streptocalyx floribundas (Martius ex Schultes F.) Mez (Bromeliaceae). Acta Bot. Brasil. 17:27-35.

Pompelli M F, D Fernandes, M P Guerra (2006) Germination of Dyckia encholirioides (Gaudichaud) Mez var. encholirioides under saline conditions. Seed Sci. Technol. 34:759-763.

Ricker M, I Ramírez-Krauss, G Ibarra-Manríquez, E Martínez, C Ramos, G González-Medellín, G Gómez-Rodríguez, J L Palacio-Prieto, H M Hernández (2007) Optimizing conservation of forest diversity: a country-wide approach in Mexico. Biodivers. Conserv.16:1927-1957.

Scatena V L, S Segecin, A I Coan (2006) Seed morphology and postseminal development of Tillandsia L. (Bromeliaceae) from the "Campus Gerais" Paraná, Southern Brazil. Braz. Arch. Biol. Techn. 49:945-951.

Schmidt L H (2007) Tropical Forest Seed. Springer-Verlag Berlin Heidelberg, New Yory, USA. $410 \mathrm{p}$.

Smith L B, R J Downs (1974) Pitcarnioideae (Bromeliaceae). Flora Neotrop.14:659-1492.

Steel R G D, J H Torrie (1985) Bioestadística: Principios y Procedimientos. McGraw-Hill Latinoamericana, S.A. Bogotá. Colombia. $622 \mathrm{p}$.

Tarré E, B B M Pires, A P M Guimaraes, L A Carneiro, R C Forzza, E Mansur (2007) Germinability after desiccation, storage and cryopreservation of seeds from endemic Encholirium Mart. Ex Schult. \& Schult. F. and Dyckia Schult. \& Schult. F. species (Bromeliaceae). Acta Bot. Brasil. 21:777-783.

Vadillo G, M Suni, A Cano (2004) Viabilidad y germinación de semillas de Puya raimondii Harms (Bromeliaceae). Rev. Peruana Biol. 11:71-78.

Vieira B C, F A O Silveira (2010) Reproductive phenology, seed germination and ex situ conservation of Pseudananas sagenarius in a semi-deciduous tropical forest fragment. Plant Species Biol. 25:214-220.

Vieira D C M, F Socolowski, M Takaki (2007) Germinação de sementes de Dyckia tuberosa (Vell.) Beer (Bromeliaceae) sob diferentes temperaturas em luz e escuro. Rev. Brasil. Bot. 30:183-188.

Wester S, G Zotz (2011) Seed comas of bromeliads promote germination and early seedling growth by wick-like water uptake. J. Trop. Ecol. 27:115-119.

Zotz G, R Asshoff (2010) Growth in epiphytic bromeliads: response to the relative supply of phosphorus and nitrogen. Plant Biol. 12:108113.

Zotz G, W Bogusch, P Hiezt, N Ketteler (2010) Growht of epiphytic bromeliads in a changing world: the effects of $\mathrm{CO} 2$, water and nutrient supply. Acta Oecol. 36:659-665. 